\title{
The associations of major foods and fibre with risk of ischaemic and haemorrhagic stroke: results from the prospective EPIC study.
}

\author{
$\underline{\text { Tammy Y.N. Tong }}{ }^{1}$, Paul N. Appleby ${ }^{1}$, Timothy J. Key ${ }^{1}$, Aurora Perez-Cornago ${ }^{1}$ and On behalf \\ of the EPIC-CVD Consortium ${ }^{2}$ \\ ${ }^{1}$ Cancer Epidemiology Unit, Nuffield Department of Population Health, University of Oxford, Richard Doll Building, \\ Old Road Campus, Oxford, United Kingdom and \\ ${ }^{2} N A$, NA, United Kingdom
}

\section{Abstract}

Introduction: The evidence of associations between individual foods and dietary fibre with subtypes of stroke (ischaemic and haemorrhagic) is not conclusive. We aimed to investigate this in a large prospective cohort.

Materials and methods: We analysed data on 418,329 men and women from the European Prospective Investigation into Cancer and Nutrition (EPIC) study. Consumption of various animal-sourced foods (red and processed meat, poultry, fish, dairy, egg), plant-sourced foods (fruit and vegetables, legumes, nuts and seeds) and dietary fibre was assessed using validated country-specific questionnaires, calibrated with 24-hour recalls. Using multivariable Cox regressions adjusted for energy intake and sociodemographic, lifestyle and physiological confounders, we estimated hazard ratios of fatal and non-fatal ischaemic, haemorrhagic and total (i.e. ischaemic, haemorrhagic and unspecified) stroke associated with calibrated increment differences in consumption of each food or dietary fibre.

Results: Over an average of 12.7 years of follow-up, we observed 4281 cases of ischaemic stroke, 1430 cases of haemorrhagic stroke, and 7378 cases of total stroke. For ischaemic stroke, lower risks were observed with higher consumption of fruit and vegetables (hazard ratio (HR); $95 \%$ confidence interval (CI) for per $200 \mathrm{~g} / \mathrm{d}$ of calibrated intake, $0.87 ; 0.82-0.93$ ) and dietary fibre (per $10 \mathrm{~g} / \mathrm{d}, \mathrm{HR}$ 0.77 ; $95 \%$ CI $0.69-0.86$ ) (p-trend $<0.001$ for both); more modest inverse associations were also observed for milk (per $200 \mathrm{~g} / \mathrm{d}, \mathrm{HR}$ $0.95 ; 95 \%$ CI $0.91-0.99$, p-trend =0.02), yogurt $($ per $100 \mathrm{~g} / \mathrm{d}$, HR 0.91; 95\% CI 0.85-0.97, p-trend $=0.004)$ and cheese $($ per $30 \mathrm{~g} / \mathrm{d}$, HR $0.88 ; 95 \%$ CI $0.81-0.97$, p-trend $=0.008$ ), while a modest positive association was observed with higher red meat consumption (per 50g/d, HR 1.14; 95\% CI 1.02-1.27, p-trend =0.02). For haemorrhagic stroke, higher risk was associated with higher egg consumption (per 20g/d, HR 1.25; 95\% CI 1.09-1.43, p-trend = 0.002). For total stroke, associations were consistent with those of both subtypes; we observed inverse associations for fruit and vegetables (HR 0.89, 95\% CI 0.85-0.93), dietary fibre (HR 0.80 , 95\% CI $0.74-0.86$ ), yogurt (HR $0.91,95 \%$ CI $0.87-0.96$ ), cheese (HR $0.88,95 \%$ CI $0.82-0.94$ ), and positive associations for red and processed meat (HR 1.18, 95\% CI 1.05-1.33) and egg (HR 1.07, 95\% CI 1.01-1.14).

Discussion: To conclude, risk of ischaemic stroke was inversely associated with consumption of fruit and vegetables, dietary fibre and dairy foods, and positively associated with red meat, while risk of haemorrhagic stroke was positively associated with egg consumption. Causality of the associations cannot be determined in this observational study.

\section{Conflict of Interest}

There is no conflict of interest. 